\title{
Identification of Atypical Diabetes Using Algorithms to Search the Electronic Medical Record
}

\author{
Verónica E. Salichs-Pérez ${ }^{1}$, Daniel Robert Hood ${ }^{2}$, Zeb Saeed ${ }^{3}$, and Carmella Evans-Molina ${ }^{4}$
}

${ }^{1}$ Ponce Health Sciences University School of Medicine; ${ }^{2}$ Regenstrief Institute; ${ }^{3}$ Indiana University School of Medicine, Division of Endocrinology and Metabolism; ${ }^{4}$ ndiana University

Center for Diabetes and Metabolic Diseases

\section{Background and Hypothesis:}

Atypical diabetes (DM) comprises phenotypically and potentially genotypically distinct forms of DM not fitting current sub-categories of classification. Understanding these rare and uncharacterized types of DM can potentially inform the application of personalized therapeutic approaches and is the emphasis of a recent $\mathrm{NIH}$-funded Consortium focused on Rare and Atypical Diabetes (RADIANT). We aimed to employ informatic-based algorithms to identify potentially atypical DM cases from electronic medical records (EMR) to identify candidates for inclusion in RADIANT.

\section{Project Methods:}

Diabetic patients were identified based on two DM diagnostic codes, treatment with anti-diabetic medication, and two diagnostic labs elevated. We filtered for patients with $\mathrm{BMI}<25$ at diagnosis and normal lipid profile. Charts were reviewed manually. Demographic and clinical data were extracted from EMR and entered into a REDCap database. SPSS was used for statistical analysis.

\section{Results:}

A total of 21,820 individuals met criteria for DM; 208 were identified as potentially atypical. Chart review was completed for 126 cases. Fifteen were deceased and removed from the final cohort. Of those remaining, $12(11 \%)$ exhibited features consistent with atypical diabetes. The remainder were classified as typical T2D. No significant differences regarding ethnicity or diabetes complication status were identified between typical and atypical cases. Demographics of atypical cases were, $66 \%$ female, and $33 \%$ male. The average age of diagnosis was $46.8 \pm 19.3 \mathrm{yrs}$. Average BMI was $23.7 \pm 4.1$. Atypical diabetes individuals were more likely to present with ketoacidosis $(p=0.01)$ exhibiting a trend towards increased insulin use $(50 \%$ vs. $14 \% ; p=0.09)$.

\section{Conclusion:}

Our study suggests that using EMR may be effective in flagging phenotypically distinct forms of DM in real world settings, associated with a high false positive rate. Data suggests this form of atypical diabetes represents a small percentage of overall DM cases $(0.05 \%)$. Future studies will focus on application of algorithms to identify other atypical diabetes subsets. 